$1987-12-01$

\title{
Definition of an Asymmetric Domain for Intercrystalline Misorientation in Cubic Materials in the Space of Euler Angles
}

Brent L. Adams

b_I_adams@byu.edu

Junwu Zhao

Follow this and additional works at: https://scholarsarchive.byu.edu/facpub

Part of the Mechanical Engineering Commons

Original Publication Citation

Acta Cryst. (1998). A44, 326-336

\section{BYU ScholarsArchive Citation}

Adams, Brent L. and Zhao, Junwu, "Definition of an Asymmetric Domain for Intercrystalline Misorientation in Cubic Materials in the Space of Euler Angles" (1987). Faculty Publications. 743.

https://scholarsarchive.byu.edu/facpub/743 


\title{
Definition of an Asymmetric Domain for Intercrystalline Misorientation in Cubic Materials in the Space of Euler Angles
}

\author{
By Junwu Zhao and Brent L. Adams \\ Department of Mechanical Engineering, Brigham Young University, Provo, Utah 84602, USA
}

\begin{abstract}
A new asymmetric domain for intercrystalline misorientation is defined in the space of Euler angles for materials exhibiting cubic ( $O_{h}$ point-group) lattice symmetry. The invariant measure for this new domain is nearly constant; this is in significant contrast to the previous domain defined by MacKenzie [Biometrika
\end{abstract}

(1958), 45, 229-240]. Distribution functions in the misorientation can now be represented with greater clarity and convenience in the new domain. A detailed theoretical analysis of special misorientations exhibiting multiplicities $m>1$ is described. It is demonstrated that all such special misorientations fall upon the surfaces separating distinct asymmetric domains. This result convincingly proves that the derived asym- 
metric domain is correct. The location of all possible coincidence site lattice boundaries for $\Sigma \leq 49$ are identified in the asymmetric domain, and their characteristic multiplicities are given.

\section{Introduction}

Quantitative descriptions of the distribution of lattice misorientation associated with grain boundaries have been studied extensively in recent years. The misorientation distribution function (MDF), first proposed by Bunge (1982) and later fully implemented by Haessner, Pospiech \& Sztwiertnia (1983) and Pospiech, Sztwiertnia \& Haessner (1986), describes the specific surface area of grain boundary associated with three parameters of misorientation. This function contains information which cannot be derived from consideration of the orientation distribution function (ODF). For example, Zhao, Adams \& Morris (1988) introduced a theoretical basis for calculating the MDF from the ODF when perfect disorder is present among the constituent grain orientations. Comparison of this theoretical MDF with the experimental one showed significant discrepancies which were attributed to orientation coherence effects. Adams (1986) and Zhao, Koontz \& Adams (1988) extended the MDF concept to include not only misorientation, but physical orientation of the grain boundary plane in a probability density function called the intercrystalline structure distribution function (ISDF). The ISDF contains all five geometrical parameters commonly associated with grain boundary structure. It is held that these new structural functions have important applications in modeling properties associated primarily with grain boundary structure; examples of recent interest in the literature include creep cavitation (Don \& Majumdar, 1986; Watanabe, 1983; Lim \& Raj, 1984), intergranular fracture (Zhao \& Adams, 1986), grain growth and recrystallization (Harase, Shimizu, Kuroki, Nakayama, Wada \& Watanabe, 1986; Harase, Shimizu \& Watanabe, 1986) and fatigue initiation (Neumann \& Toennessen, 1987).

The description of misorientation distributions in cubic materials has suffered from a serious problem of representation. When the axis-angle representation of misorientation is chosen, a serious nonlinearity exists in the asymmetric domain of representation. (The asymmetric domain is here defined to be the minimum compact subvolume in a space of the chosen misorientation parameters required to contain each physically distinct misorientation once and only once). MacKenzie $(1958,1964)$ derived a particular asymmetric domain of misorientation based on the requirement of a minimum absolute rotation angle. This domain is problematical, however, since the invariant measure associated with the axis-angle description contains a term in the square of the sine of the rotation angle which tends to zero as the rotation angle approaches zero. Consequently distribution functions defined over the domain of MacKenzie tend to be ambiguous for small values of the rotation angle since the axis of rotation is no longer precisely defined.

The other common method of representation for cubic materials, that of Euler angles, has been even more problematical since an asymmetric domain was not previously defined. Representations in the space of Euler angles have typically been given over a larger domain such that specific misorientations occur with non-uniform multiplicity. Such non-uniformity is particularly troublesome in the interpretation of the distribution function.

In $\S 2$ a new asymmetric domain for misorientation in cubic materials ( $O_{h}$ point-group symmetry) is derived in the space of Euler angles. An important advantage of the new domain is that the invariant measure associated with the space of Euler angles is nearly constant over its volume; the problems associated with the axis-angle domain of MacKenzie are consequently eliminated. $\S 3$ gives a rigorous treatment of multiplicity of misorientation in the space of Euler angles based upon certain group- and matrixtheoretical concepts. It is illustrated that all misorientations of multiplicity greater than 1 lie upon the boundaries of the new asymmetric domain as required. Finally, in $\S 4$, a useful connection is constructed between the coincidence-site-lattice (CSL) theory and the new domain. The locations of all CSL boundaries for $\Sigma \leq 49$ are located in the domain. These developments provide a comprehensive basis for representing and interpreting misorientation distributions in the space of Euler angles.

\section{Derivation of an asymmetric domain}

The derivation is principally concerned with intercrystalline misorientations in cubic materials exhibiting $O_{h}(m \overline{3} m)$ point-group symmetry in the crystal lattice. We will include the center of symmetry of $O_{h}$ at the outset by considering only misorientations of proper character (determinant of positive 1). With this restriction enforced in the beginning, further consideration of cubic crystal symmetry can be restricted to the elements of the $O(432)$ point group. With reference to Fig. 1, consider lattice-fixed orthogonal coordinate systems $K_{A}$ and $K_{B}$ associated with grains $A$ and $B$ which join at a common boundary. The axes of $K_{A}$ and $K_{B}$ are parallel to $\langle 100\rangle$ directions in the lattice. Misorientation $g$ is defined to be the proper rotation which transforms the reference system $K_{A}$ into $K_{B}$. Specifically, $g$ is the $3 \times 3$ matrix of direction cosines connecting the two coordinate frames. With reference to Fig. 1, the $i$ th row and $j$ th column $(i, j=1,2,3)$ of rotation $g, g_{i j}$, is

$$
g_{i j}=\cos \left\langle X_{B}^{i}, X_{A}^{j}\right\rangle
$$


where $\left\langle X_{B}^{i}, X_{A}^{j}\right\rangle$ denotes the angle between the $j$ th coordinate axis associated with $K_{A}$ and the $i$ th coordinate axis associated with $K_{B}$. The misorientation $g$ is given in terms of three consecutive right-handed rotations $\varphi_{1}, \phi, \varphi_{2}$ as defined in Fig. 1. $\varphi_{1}$ is a rotation about $X_{A}^{3} \cdot \phi$ is a rotation about $X_{A}^{1}$, and the last rotation $\varphi_{2}$, is about the $X_{B}^{3}$ axis. The matrix elements of the misorientation rotation, $g_{i j}$, are

$$
\begin{aligned}
& g_{11}=\cos \varphi_{1} \cos \varphi_{2}-\sin \varphi_{1} \sin \varphi_{2} \cos \phi, \\
& g_{12}=\sin \varphi_{1} \cos \varphi_{2}+\cos \varphi_{1} \sin \varphi_{2} \cos \phi, \\
& g_{13}=\sin \varphi_{2} \sin \phi, \\
& g_{21}=-\cos \varphi_{1} \sin \varphi_{2}-\sin \varphi_{1} \cos \varphi_{2} \cos \phi, \\
& g_{22}=-\sin \varphi_{1} \sin \varphi_{2}+\cos \varphi_{1} \cos \varphi_{2} \cos \phi, \\
& g_{23}=\cos \varphi_{2} \sin \phi, \\
& g_{31}=\sin \varphi_{1} \sin \phi, \\
& g_{32}=-\cos \varphi_{1} \sin \phi, \\
& g_{33}=\cos \phi .
\end{aligned}
$$

We shall subsequently use the notation $g=\left(\varphi_{1}, \phi, \varphi_{2}\right)$ to represent misorientation in compact form.

Misorientations can be thought of in terms of an abstract space in Euler angles. The triplet of values $\left(\varphi_{1}, \phi, \varphi_{2}\right)$ defines a point in this three-dimensional space spanned by three axes representing the continuous variation in the Euler angles, $\varphi_{1}, \phi$ and $\varphi_{2}$. In the absence of crystal symmetry (beyond the center of symmetry), an asymmetric subvolume defined by
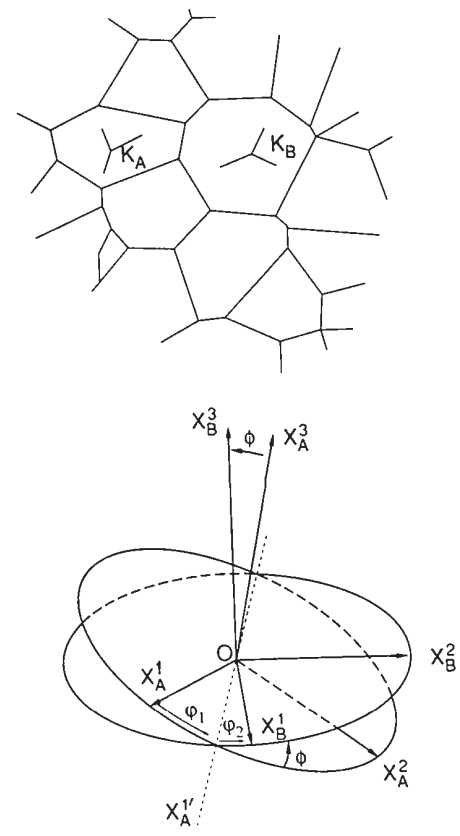

Fig. 1. Euler angles $\varphi_{1}, \phi, \varphi_{2}$, which rotate crystal frame $A$ into crystal frame $B$.
Table 1. The 25 possible symmetry elements of the $O$ group

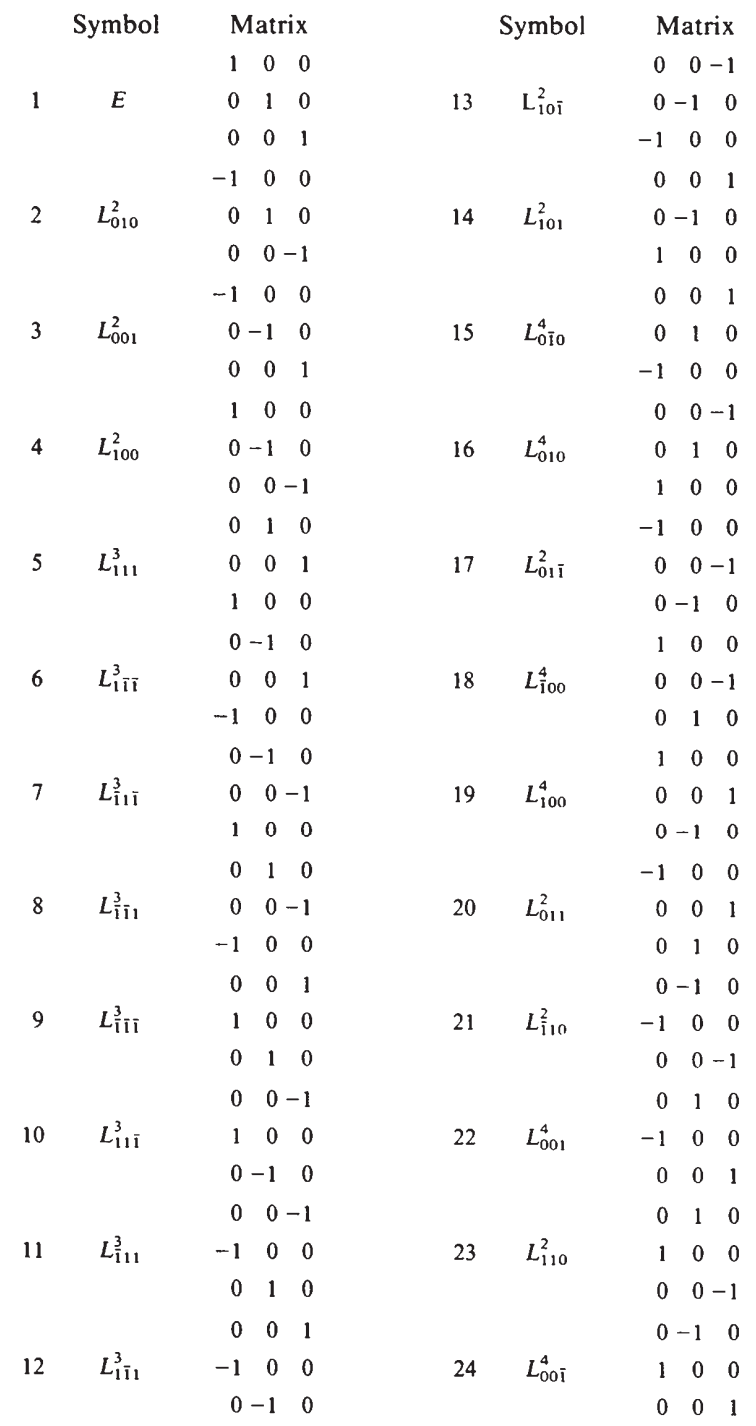

the relations $0 \leq \varphi_{1} \leq 2 \pi, 0 \leq \phi \leq \pi$, and $0 \leq \varphi_{2} \leq 2 \pi$ confines all physically distinct misorientations. When crystal symmetry is present additional constraints are prescribed which further limit the size of the asymmetric domain. Here we use the notation of Pospiech, Gnatek \& Fichtner (1974) and Hansen, Pospiech \& Lucke (1978) for the elements of the crystal point group. Let $L_{[u v w]}^{n}$ represent the matrix for an $n$-fold rotation of the crystal coordinate frame about the $[u v w]$ crystal direction. All such elements are restricted to be members of the $O$ (432) crystal point group which contains 24 members. (Table 1 lists the elements of the $O$ group.) Crystal symmetry associated with grain $A$ requires that misorientation $g^{\prime}=g L_{[u v w]}^{n}$ is physically indistinguishable from $g$ when $L_{[u v w]}^{n} \in$ $O$. Crystal symmetry associated with grain $B$ requires 
that the misorientation $g^{\prime \prime}=L_{[r s t]}^{m} g$ is indistinguishable from $g$ when $L_{[r s t]}^{m} \in O$. It follows that the general condition for equivalency is given by

$$
g^{e}=L_{[r s t]}^{m} g L_{[u v w]}^{n},
$$

where $L_{[r s t]}^{m}, L_{[u w w]}^{n} \in O$, and therefore $g^{e}$ is equivalent to $g$.

Pospiech et al. (1974) have given a complete analysis of 'lower' or 'non-cubic' symmetry groups and the consequent reductions in the asymmetric domain in Euler space. Here we will just briefly indicate that careful consideration of the equivalency relationships

$$
g^{e}=E g L_{[001]}^{4}=L_{[001]}^{4} g E=L_{[110]}^{2} g L_{[001]}^{2}
$$

permits the asymmetric domain to be reduced to the subvolume defined by the relations $0 \leq \varphi_{1} \leq \pi / 2,0 \leq$ $\phi \leq \pi / 2$, and $0 \leq \varphi_{2} \leq \pi / 2$. Consideration of the threefold rotations $L_{[u v w]}^{3}$ introduces nonlinear relationships which further constrain the necessary size of the asymmetric domain. These are now considered in detail.

Consider the effect of twofold rotations about $\langle 110\rangle$ axes on both coordinate systems:

$$
g^{e}=L_{[\overline{1} 10]}^{2} g L_{[110]}^{2} .
$$

This equivalence requires that

$$
\left(\varphi_{1}, \phi, \varphi_{2}\right)^{e}=\left(\pi / 2-\varphi_{1}, \phi, \pi / 2-\varphi_{2}\right) .
$$

We shall require our distribution function to be invariant with respect to the interchange of the two crystallites $\left(K_{A} \rightarrow K_{B}, K_{B} \rightarrow K_{A}\right)$. Formally this requires that $g^{e}=g^{-1}=g^{T}$ (since $g$ is unitary orthogonal), or

$$
\left(\varphi_{1}, \phi, \varphi_{2}\right)^{e}=\left(\pi-\varphi_{2}, \phi, \pi-\varphi_{1}\right) .
$$

As a consequence of the equivalence of misorientation under the right- and left-hand operations of fourfold rotation elements, $L_{[u v w]}^{4}$,

$$
\left(\varphi_{1}, \phi, \varphi_{2}\right)^{e}=\left(\varphi_{1}+n \pi / 2, \phi, \varphi_{2}+m \pi / 2\right),
$$

where $n$ and $m$ are positive and negative integers. Combining (8) with (7) for $n=m=-1$, we require

$$
\left(\varphi_{1}, \phi, \varphi_{2}\right)^{e}=\left(\pi / 2-\varphi_{2}, \phi, \pi / 2-\varphi_{1}\right) .
$$

Further, combining (6) and (9), we require

$$
\left(\varphi_{1}, \phi, \varphi_{2}\right)^{e}=\left(\varphi_{2}, \phi, \varphi_{1}\right)
$$

Equivalences (9) and (10) define two mirror planes, as illustrated in Fig. 2. A reduced asymmetric region can be selected, as shown by the shading in Fig. 2, by the relations

$$
\begin{gathered}
0 \leq \varphi_{1} \leq \varphi_{2} \leq \pi / 2, \quad 0 \leq \phi \leq \pi / 2, \\
\varphi_{1}+\varphi_{2} \leq \pi / 2 .
\end{gathered}
$$

Next, consider the actions of threefold rotation elements, $L_{[u v w]}^{3}$, on $K_{A}$ and $K_{B}$. The right-hand operation of such elements switches columns in the misorientation matrix $g$. The left-hand operation of threefold rotation elements switches rows in $g$. The combined effect is the possibility of switching any element of matrix $g$ to any other position in the matrix. In combination with other twofold and fourfold symmetry elements, not only can the position be arbitrarily changed, but also the sign.

We shall require that $\phi$ be maximized in the range $0 \leq \phi \leq \pi / 2$. This is equivalent to minimizing $\cos \phi$ over this range. Since $g_{33}=\cos \phi$, and as a consequence of the position and sign-switching properties asociated with the threefold symmetry elements, we must require that $g_{33}=\cos \phi$ be less than (or equal to) the positive value of every other element of $g$. Since the sine and cosine of every possible angle in the reduced domain of Fig. 2 are positive, this requires that

$\cos \phi \leq\left|\cos \varphi_{1} \cos \varphi_{2}-\sin \varphi_{1} \sin \varphi_{2} \cos \phi\right|=\left|g_{11}\right|$,

$\cos \phi \leq\left|-\sin \varphi_{1} \sin \varphi_{2}+\cos \varphi_{1} \cos \varphi_{2} \cos \phi\right|=\left|g_{22}\right|$,

$\cos \phi \leq \sin \varphi_{1} \cos \varphi_{2}+\cos \varphi_{1} \sin \varphi_{2} \cos \phi=g_{12}$,

$\cos \phi \leq \cos \varphi_{1} \sin \varphi_{2}+\sin \varphi_{1} \cos \varphi_{2} \cos \phi=-g_{21}$,

$\cos \phi \leq \sin \varphi_{1} \sin \phi=g_{31}$,

$\cos \phi \leq \cos \varphi_{1} \sin \phi=-g_{32}$,

$\cos \phi \leq \sin \varphi_{2} \sin \phi=g_{13}$,

$\cos \phi \leq \cos \varphi_{2} \sin \phi=g_{23}$.

For angles satisfying the inequalities of (11) (shaded region in Fig. 2), (12) is equivalent to

$\cos \phi \leq \cos \varphi_{1} \cos \varphi_{2} /\left(1+\sin \varphi_{1} \sin \varphi_{2}\right)$,

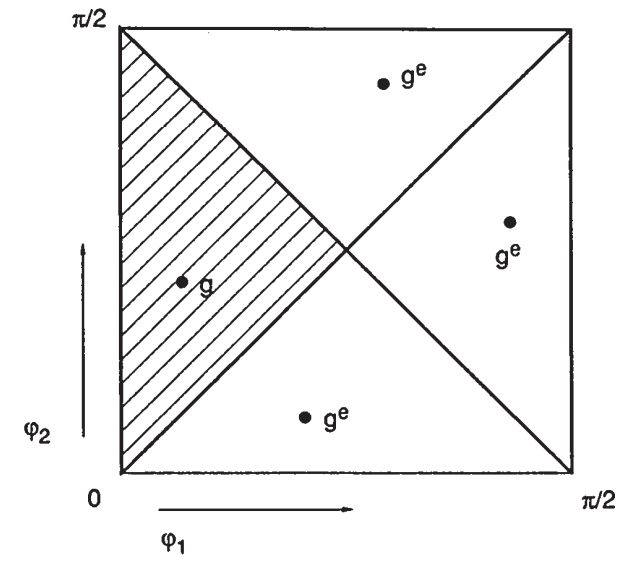

Fig. 2. Two mirror planes introduced by crystal symmetry and the inverse property. The symbol $g^{e}$ denotes the physically equivalent misorientation of $g$. 
and (13) is equivalent to

$$
\cos \phi \leq \sin \varphi_{1} \sin \varphi_{2} /\left(1+\cos \varphi_{1} \cos \varphi_{2}\right) \text {. }
$$

However, since $0 \leq\left(\varphi_{1}+\varphi_{2}\right) \leq \pi / 2, \sin \varphi_{1} \sin \varphi_{2} \leq$ $\cos \varphi_{1} \cos \varphi_{2}$; also the inequality (21) is more restrictive than (20). As a consequence it will only be necessary to consider (21). The inequality (14) is equivalent to

$$
\cos \phi \leq \sin \varphi_{1} \cos \varphi_{2} /\left(1-\cos \varphi_{1} \sin \varphi_{2}\right),
$$

and (15) is equivalent to

$$
\cos \phi \leq \cos \varphi_{1} \sin \varphi_{2} /\left(1-\sin \varphi_{1} \cos \varphi_{2}\right) .
$$

Since $\varphi_{1} \leq \pi / 4, \sin \varphi_{1} \leq \cos \varphi_{1}$ and $1+\cos \varphi_{1} \cos \varphi_{2} \geq$ $1-\sin \varphi_{1} \cos \varphi_{2}$, the inequality (23) is included in (21). Because $\varphi_{1} \leq \pi / 4,0 \leq \varphi_{1} \leq \varphi_{2} \leq \pi / 2$ and $\left(\varphi_{1}+\right.$ $\left.\varphi_{2}\right) \leq \pi / 2$, relations (17), (18) and (19) are included in (16) which can be rewritten as

$$
\cos \phi \leq \sin \varphi_{1} /\left(1+\sin ^{2} \varphi_{1}\right)^{1 / 2} \text {. }
$$

Relation (24) is not as restrictive as (21) and can be included in it. To see this, note that $1 / \sin \varphi_{2}+$ $\sin \varphi_{1} \geq 1+\sin ^{2} \varphi_{1} \geq\left(1+\sin ^{2} \varphi_{1}\right)^{1 / 2}$ and therefore $\sin \varphi_{1} /\left(1 / \sin \varphi_{2}+\sin \varphi_{1}\right) \leq \sin \varphi_{1} /\left(1+\sin ^{2} \varphi_{1}\right)^{1 / 2}$.

Consequently

$$
\begin{gathered}
\sin \varphi_{1} \sin \varphi_{2} /\left(1+\sin \varphi_{1} \sin \varphi_{2}\right) \\
\leq \sin \varphi_{1} /\left(1+\sin ^{2} \varphi_{1}\right)^{1 / 2} .
\end{gathered}
$$

Since $\sin \varphi_{1} \sin \varphi_{2} \leqslant \cos \varphi_{1} \cos \varphi_{2}$ it is clear that

$$
\begin{gathered}
\sin \varphi_{1} \sin \varphi_{2} /\left(1+\cos \varphi_{1} \cos \varphi_{2}\right) \\
\leq \sin \varphi_{1} /\left(1+\sin ^{2} \varphi_{1}\right)^{1 / 2},
\end{gathered}
$$

which means that the inequality (24) is included in (21).

To summarize the results to this point, we have shown that the asymmetric region is bounded by the inequalities of (11), (21) and (22). To complete the derivation we will now demonstrate that $(22)$ is contained within (21). This is immediately obvious when $\varphi_{2} \leq \pi / 4$. For $\varphi_{2} \geq \pi / 4$ and arbitrary choices of $\varphi_{1}$ and $\varphi_{2}$ satisfying relation (11) we must show that

$$
\frac{\sin \varphi_{1} \cos \varphi_{2}}{1-\cos \varphi_{1} \sin \varphi_{2}} \geq \frac{\sin \varphi_{1} \sin \varphi_{2}}{1+\cos \varphi_{1} \cos \varphi_{2}}
$$

Rearrangement of this relation leads to the equivalent expression

$$
\cos \varphi_{1} \geq \sqrt{2} \sin \left(-\pi / 4+\varphi_{2}\right) .
$$

Since $\varphi_{2} \geq \pi / 4 \geq \varphi_{1}$ and $\varphi_{1}+\varphi_{2} \leq \pi / 2$, let us rewrite $\varphi_{2}$ as $\varphi_{2}=\alpha+\pi / 4$ where $0 \leq \alpha \leq \pi / 4$. Then $\varphi_{1} \leq$ $\pi / 4-\alpha$ and $\cos \varphi_{1} \geq \cos (\pi / 4-\alpha)=\sin (\pi / 4+\alpha)$. The inequality $(28)$ is contained within the relation

$$
\sin (\pi / 4+\alpha) \geq \sqrt{2} \sin \alpha,
$$

which is equivalent to the relation $\cos (\pi / 4+\alpha) \geq 0$, and therefore (29) is true when $0 \leq \alpha \leq \pi / 4$. From the above we conclude that the asymmetric domain for misorientation in the space of Euler angles is bounded by the relations

$$
0 \leq \cos \phi \leq \sin \varphi_{1} \sin \varphi_{2} /\left(1+\cos \varphi_{1} \cos \varphi_{2}\right)
$$

and

$$
0 \leq \varphi_{1} \leq \varphi_{2} \leq \pi / 2, \phi \geq 0, \varphi_{1}+\varphi_{2} \leq \pi / 2 \text {. }
$$

This is depicted graphically in Fig. 3.

The subvolume of the asymmetric domain is required to be equal to $8 \pi^{2} / 1152=\pi^{2} / 144$ (MacKenzie, 1964). It is immediately confirmed that this volume is

$$
\int_{0}^{\pi / 4} \int_{\varphi_{1}}^{\pi / 2-\varphi_{1} \sin \varphi_{1} \sin \varphi_{2} /\left(1+\cos \varphi_{1} \cos \varphi_{2}\right)} \int_{0}^{2} \mathrm{~d} \zeta \mathrm{d} \varphi_{2} \mathrm{~d} \varphi_{1}=\pi^{2} / 144,
$$

where $\zeta=\cos \phi$.

\section{Multiplicity of misorientation in the space of Euler angles}

It is a property of the asymmetric domain that every interior point of the asymmetric subvolume represents a physically distinct misorientation. A consequence of symmetry is that every interior point $g$ will map to 1151 other equivalent points, each one residing in another equivalent asymmetric domain. It is possible to define all of these domains mathematically, but the process is tedious and has not been undertaken by the authors. The boundaries separating these asymmetric subvolumes contain points of two types. The first type consists of misorientation points which always map to other points also lying on the separating surfaces; these points must never map into the interior under the combined action of symmetry elements. Boundary misorientations of this first type

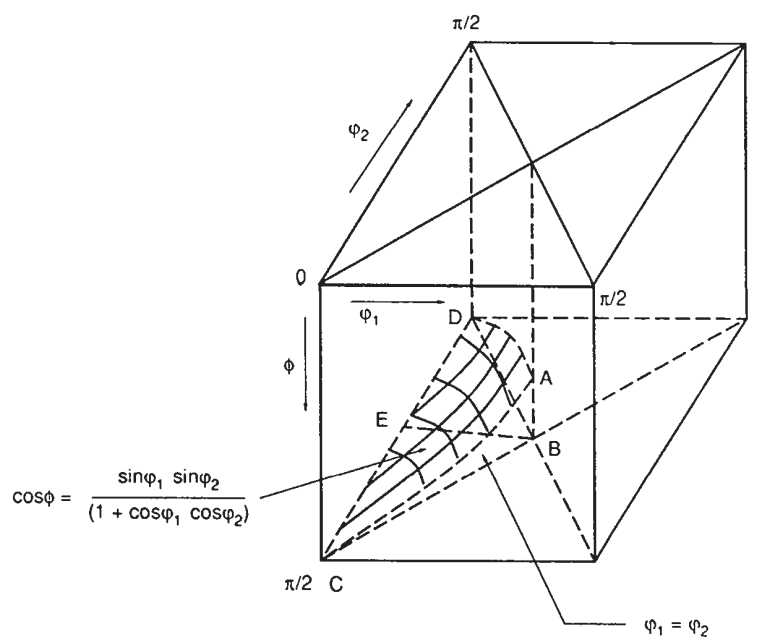

Fig. 3. The asymmetric region defined by the subvolume $A B C D$. 
map to 1151 other boundary points under the action of the symmetry elements. The second type of boundary misorientation has a special property, called multiplicity, such that it maps back upon itself under specified combinations of the symmetry elements. Thus if $m$ is the multiplicity number, such points occur not 1152 times in Euler space, but 1152/m times. Misorientations of this type must always be on the boundaries separating asymmetric subvolumes. Demonstration that all such points lie on the surfaces of the asymmetric domain given in (30) and (31) is convincing confirmation of its correctness.

To this end we shall classify all possible misorientations in Euler space which exhibit multiplicity under the combined right- and left-handed action of elements of the $O$ crystal point group on $K_{A}$ and $K_{B}$. It is then demonstrated that all such special misorientations are found upon the defined surfaces of the chosen asymmetric subvolume. The reader may not be familiar with theoretical aspects of the group theory. The book by Hamermesh (1964) is recommended if assistance is required. We shall adopt an abbreviated notation for the elements of the $O$ group; let $L_{i}, i=1,2, \ldots, 24$ represent any one of the 24 elements of the $O$ group listed in Table 1. Further, define $L_{i j}$ to be the left- and right-hand pair of symmetry elements $\left(L_{i}, L_{j}\right)$ operating on misorientation $g$ such that $g^{e}=L_{i} g L_{j}=L_{i j} g$. In the discussion which follows it will be necessary also to consider the equivalence of $g$ with respect to interchange of $K_{A}$ and $K_{B}$; initially, however, only 576 symmetry operations are considered.

Formally, the multiplicity number $m$ is defined as the number of distinct pairs of symmetry elements $L_{i j}$ which can be found such that

$$
g=L_{i j} g
$$

for a particular chosen $g$.

We now set forth five theorems, and a sketch of their proofs, which are necessary for the classification of misorientations satisfying the multiplicity relation (33).

\subsection{Derivation of fundamental theorems}

Theorem 1. All of the operators $L_{i j}$ satisfying the relationship $L_{i j} g=g$ form a group $Q_{g}$.

Proof of theorem 1.

(1) The elements $L_{i}$ and $L_{j}$ belong to the $O$ pointsymmetry group; consequently there exist only 576 possible elements $L_{i j}$ which can be formed. Consider the set $Q=\left\{L_{i j}\right\}$ such that every element of the set satisfies the relationship $L_{i j} g=g$.

(2) If $L_{r s}$ and $L_{t u} \in Q$ then $L_{r s} L_{t u} g=L_{r s} g=g$. Therefore the set $Q$ is closed under the operation $L_{r s} L_{t u}$.

(3) The consecutive operation of elements $L_{i j} \in Q$ is associative. That is, if $L_{r s}, L_{t u}$ and $L_{i j} \in Q$, then $\left(L_{r s} L_{t u}\right) L_{i j}=L_{r s}\left(L_{t u} L_{i j}\right)$.
(4) The set $Q$ must contain the identity element $L_{i j}=(E, E)$.

(5) The set $Q$ will contain, for every $L_{i j} \in Q$, the inverse of $L_{i j}$. Since $L_{i j} g=L_{i} g L_{j}=g$ it follows that $L_{i}^{-1} g L_{j}^{-1}=g$. Since $L_{i}, L_{j}, L_{i}^{-1}$ and $L_{j}^{-1} \in O, L_{i j}^{-1}=$ $\left(L_{i}^{-1}, L_{j}^{-1}\right) \in Q$.

(6) It follows from (2), (3), (4) and (5) that set $Q$ is a group, hereafter referred to as $Q_{g}$ to suggest that, for each misorientation $g$ exhibiting multiplicity $m>$ 1 , there will in general exist a different group $Q_{g}$. The order of group $Q_{g}$ will be the multiplicity, $m$, associated with misorientation $g$.

Theorem 2. If $L_{i j} \in Q_{g}$ then elements $L_{i}$ and $L_{j}$ forming $L_{i j}$ must have the same set of eigenvalues.

Proof of theorem 2.

If $L_{j} \in Q_{g}$ then $L_{i}=g L_{j}^{-1} g^{-1}$. It follows that $L_{i}$ and $L_{j}^{-1}$ are similar matrices and therefore must have the same set of eigenvalues.

(2) Every element of the $O$ group can be classified according to its eigenvalues. (Associated with the eigenvalues $1,1,1$ is the identity element $E$. Associated with the eigenvalues $-1,-1,1$ are all of the nine twofold rotation elements of the form $L_{110}^{2}$ and $L_{100}^{2}$. Associated with the eigenvalues $1, i,-i$ are the six fourfold rotation elements. And associated with the eigenvalues $1,-\frac{1}{2}+i \sqrt{\frac{3}{2}},-\frac{1}{2}-i \sqrt{\frac{3}{2}}$ are the eight threefold rotation elements.) Thus elements of the same order of rotation have the same eigenvalues.

(3) The inverse of $L_{j}, L_{j}^{-1}$, has the same order as $L_{j}$ and therefore the same eigenvalues. This can be proven as follows: Let $n$ be the order of $L_{j}$. By definition $L_{j}^{n}=E$. Multiply both sides of this equation by $\left(L_{j}^{n}\right)^{-1}=\left(L_{j}^{-1}\right)^{n}$ to form $E=\left(L_{j}^{-1}\right)^{n}$. Therefore the order of $L_{j}^{n}$ is equal to that of $L_{j}$.

One important consequence of theorem 2 is in limiting the number of pairs $\left(L_{i}, L_{j}\right)$ which must be considered in forming group $Q_{g}$. Elements of the form $\left(L_{u v w}^{n}, L_{r s t}^{m}\right)$ are prohibited for $n \neq m$. In other words, the order of $L_{i j}$ must be equivalent to the order of the elements $L_{i}$ and $L_{j}$ from which it is formed. Only orders 1, 2, 3 and 4 are possible. Another consequence of theorem 2 derives from consideration of Lagrange's theorem (Hamermesh, 1964). The order of any element of the finite group $Q_{g}$ must be a divisor of the order of $Q_{g}$. Therefore the order of $Q_{g}$ cannot be 5, 7, 11, 13 etc.

Theorem 3. The maximum possible order of $Q_{g}$ is 24.

Proof of theorem 3.

(1) Assume that $g$ exists such that $L_{i j} g=g$. From the definition of $L_{i j}$ this implies that for specified $L_{j}$ there exists a unique $L_{i}$ given by $L_{i}=g L_{j}^{-1} g^{-1}$.

(2) Since $L_{j} \in O$ there exist only 24 possible elements $L_{j}$. Consequently a maximum of 24 elements $L_{i j}$ can exist for any group $Q_{g}$. Thus the order of $Q_{g}$ is $\leq 24$. 
Theorem 4. If the misorientation $g$ is known such that $L_{i} g L_{j}=g$, and $L_{k}, L_{m} \in O$ have the same order as $L_{i}$ and $L_{j}$, and if the axis of rotation of $L_{k}$ is orthogonal to the axis of $L_{i}$ and, likewise, if the axis of rotation of $L_{m}$ is orthogonal to that of $L_{j}$, then the matrix form of $g^{\prime}$ is known such that $L_{k} g^{\prime} L_{m}=g^{\prime}$.

Proof of theorem 4.

(1) If $L_{i}$ and $L_{k}$ are of the same order, and the respective axes of their rotations are orthogonal then there exists $L_{h} \in O$ such that $L_{i}=L_{h} L_{k} L_{h}^{-1}$. (This can easily be understood, geometrically, as $L_{h}^{-1}$ rotates the current coordinate system to a new position such that the rotation axis associated with $L_{i}$ becomes the rotation axis of $L_{k}$, and $L_{h}$ rotates the coordinate system back again after rotation $L_{k}$ is performed. All required rotations of this sort are included in the $O$ point group.)

(2) If $L_{i}=L_{h} L_{k} L_{h}^{-1}$ and $L_{j}=L_{p} L_{m} L_{p}^{-1}$, then $L_{h} L_{k} L_{h}^{-1} g L_{p} L_{m} L_{p}^{-1}=g$, or $L_{k}\left(L_{h}^{-1} g L p\right) L_{m}=L_{h}^{-1} g L_{p}$. Thus $g^{\prime}$ has the form of $L_{h}^{-1} g L_{p}$. (Clearly, the exact misorientation, $g^{\prime}$, which satisfies the relationship $L_{k} g^{\prime} L_{m}=g^{\prime}$ is not unique, but the form of $g^{\prime}$ is unique.)

The importance of theorem 4 is now explained. Note that all physically equivalent orientations $g^{e}=$ $L_{i} g L_{j}$ must have identical multiplicities, $m$. Consider the cyclic group $A$ formed from a particular generator $L_{i j}$. Suppose that $g$ is known such that $L_{i j} g=g$. All elements of the group $A$ satisfy the same relationship. Suppose that we consider another cyclic group $B$ of the same order as $A$ such that the axes of rotation associated with elements of $B$ are orthogonal on the left hand and the right hand to the axes of rotation for elements of $\boldsymbol{A}$. From theorem $5 \mathrm{~g}^{\prime}$ is known to be physically equivalent to $g$ where $g^{\prime}$ satisfies the relation $L_{i j}^{\prime} g^{\prime}=g^{\prime}$ and $L_{i j}^{\prime} \in B$. It is therefore only necessary to find one misorientation $g$ satisfying the relation $L_{i j} g=g$ for a single element $L_{i j} \in A$ to treat comprehensively all elements of all possible cyclic groups of the same order, so long as the axes of rotation are orthogonal.

Theorem 5. For a group $Q_{g}$, if $L_{i j} \in Q_{g}$, then $Q_{g}$ contains the cyclic group $\left\{L_{i j}^{n} \mid n\right.$ is an integer $\}$.

Proof of theorem 5. This theorem is fundamental to group theory, and is proven in all elementary texts on the subject (Fraleigh, 1982).

A detailed study of the $O$ group and the groups $Q_{g}$ which can be formed from elements of the $O$ group reveals that there are seven types of cyclic groups which mut be considered. These cyclic groups are generated from elements of the type $(E, E)$, $\left(L_{100}^{2}, L_{001}^{2}\right), \quad\left(L_{111}^{3}, L_{111}^{3}\right),\left(L_{110}^{2}, L_{110}^{2}\right), \quad\left(L_{100}^{2}, L_{110}^{2}\right)$, $\left(L_{110}^{2}, L_{100}^{2}\right)$ and $\left(L_{100}^{4}, L_{100}^{4}\right)$. In combination with theorem 4 , it is now clear that these seven distinct elements identify all possible misorientations $g$ associated with all possible cyclic groups.

\subsection{Detailed consideration of the multiplicity relations}

We now consider the form of misorientation $g$ required to satisfy the multiplicity relationship (33) for each of the seven types of cyclic groups previously identified.

From the generator $L_{i j}=(E, E)$ all misorientations satisfy the multiplicity relation; thus no constraint is imposed.

For the generator of type $L_{i j}=\left(L_{100}^{2}, L_{100}^{2}\right)$ or the generator $L_{i j}=\left(L_{100}^{4}, L_{100}^{4}\right)$ it is required that $g$ have the form

$$
g=\left(\begin{array}{ccc}
1 & 0 & 0 \\
0 & \cos \omega & \sin \omega \\
0 & -\sin \omega & \cos \omega
\end{array}\right),
$$

where $\omega$ is variable. The multiplicity of these misorientations is 4 . The form of misorientation associated with this group is interpreted to be due to rotations around the $\langle 100\rangle$ axes.

For the generator $L_{i j}=\left(L_{110}^{2}, L_{110}^{2}\right)$, it is required that

$$
g=\left(\begin{array}{ccc}
\frac{1+\cos \omega}{2} & \frac{1-\cos \omega}{2} & -\frac{1}{\sqrt{2}} \sin \omega \\
\frac{1-\cos \omega}{2} & \frac{1+\cos \omega}{2} & \frac{1}{\sqrt{2}} \sin \omega \\
\frac{1}{\sqrt{2}} \sin \omega & -\frac{1}{\sqrt{2}} \sin \omega & \cos \omega
\end{array}\right)
$$

Here the multiplicity is 2 . This form of misorientation can be interpreted to derive from rotations around $\langle 110\rangle$ axes.

When $L_{i j}=\left(L_{111}^{3}, L_{\overline{1} 1 \overline{1}}^{3}\right), g$ must be

$$
g=\left(\begin{array}{ccc}
\frac{1}{3}(2 \cos \omega+1) & \frac{1}{3}(1-\cos \omega)+\frac{1}{\sqrt{3}} \sin \omega & \frac{1}{3}(1-\cos \omega)-\frac{1}{\sqrt{3}} \sin \omega \\
\frac{1}{3}(1-\cos \omega)-\frac{1}{\sqrt{3}} \sin \omega & \frac{1}{3}(2 \cos \omega+1) & \frac{1}{3}(1-\cos \omega)+\frac{1}{\sqrt{3}} \sin \omega \\
\frac{1}{3}(1-\cos \omega)+\frac{1}{\sqrt{3}} \sin \omega & \frac{1}{3}(1-\cos \omega)-\frac{1}{\sqrt{3}} \sin \omega & \frac{1}{3}(2 \cos \omega+1)
\end{array}\right) .
$$

Misorientations of this type have multiplicity of 3 . Such misorientations have the form associated with rotations about $\langle 111\rangle$ axes.

For $L_{i j}=\left(L_{110}^{2}, L_{100}^{2}\right), g$ must have the form

$$
g=\left(\begin{array}{ccc}
-\sin \omega & \cos \omega & 0 \\
\frac{1}{\sqrt{2}} \cos \omega & \frac{1}{\sqrt{2}} \sin \omega & \frac{1}{\sqrt{2}} \\
\frac{-1}{\sqrt{2}} \cos \omega & -\frac{1}{\sqrt{2}} \sin \omega & \frac{1}{\sqrt{2}}
\end{array}\right) .
$$

The multiplicity here is 2 .

Note that if $L_{i} g L_{j}=g$ then $L_{j}^{-1} g^{-1} L_{i}^{-1}=g^{-1}$ and since $L_{110}^{2}{ }^{-1}=L_{110}^{2}$ and $L_{100}^{2}{ }^{-1}=L_{100}^{2}$ it follows that for $L_{i j}=\left(L_{100}^{2}, L_{110}^{2}\right)$ the form of $g$ must be the inverse 
(transpose) of (37):

$$
g=\left(\begin{array}{ccc}
-\sin \omega & \frac{1}{\sqrt{2}} \cos \omega & \frac{-1}{\sqrt{2}} \cos \omega \\
\cos \omega & \frac{1}{\sqrt{2}} \sin \omega & \frac{-1}{\sqrt{2}} \sin \omega \\
0 & \frac{1}{\sqrt{2}} & \frac{1}{\sqrt{2}}
\end{array}\right) .
$$

Again the multiplicity is 2. Misorientations of the form given in (37) and (38) cannot be interpreted simply in terms of a unique type of rotation axis.

It is clear that for every order of cyclic group only one adjustable variable, $\omega$, remains. Since by theorem 5 for any $g$ the group $Q_{g}$ must include as a minimum the cyclic subgroups which can be formed from its elements, it follows that $Q_{g}$ must contain the restrictions placed upon these cyclic subgroups as a minimal constraint. If the order of $Q_{g}$ is greater than the maximum order of its cyclic subgroups then further constraints on $g$ are required. However, since only one adjustable variable remains, these further constraints must operate solely upon the variable $\omega$. In other words, the form of the misorientation $g$ defined by the constraint equations (34) through (38) can be subject to further constraint (higher multiplicity) for specific choices of $\omega$. It is therefore not necessary to consider all possible groups $Q_{g}$, but rather only the further constraints which can be imposed upon the variable $\omega$ in (34) through (38) to increase the multiplicity of $g$.

The constraints in (34)-(38) are curved lines in the space of Euler angles. These lines have been identified to lie upon the surface of the asymmetric domain derived in the previous section. These lines are shown in Fig. 3. The points in Fig. 3 are identified in terms of their Euler angles, $\left(\varphi_{1}, \phi, \varphi_{2}\right)$, as follows: $A=$ $\left(\pi / 4, \arccos \frac{1}{3}, \pi / 4\right), \quad B=(\pi / 4, \pi / 2, \pi / 4), \quad C=$ $(0, \pi / 2,0), \quad D=(0, \pi / 2, \pi / 2), \quad E=(0, \pi / 2, \pi / 4)$. The line associated with multiplicity of 4 [(34)] is the straight-line segment $C D$. Associated with (35) is the straight-line segment $A B$ and the curved-line segment $A C$. Equation (36) is associated with multiplicity 3 and the curved-line segment $A D$. Equation (37), associated with a multiplicity of 2 , is represented by the straight-line segment $B E$. Equation (38) is found to be equivalent to (37) when the misorientation inverse property of (7) is invoked. Clearly, all possible points of multiplicity $m>1$ are located on the boundary of the defined asymmetric domain.

Theorem 3 requires that the maximum order of $Q_{g}$ is 24 . There are 24 possible misorientations $g$ with multiplicity of 24 . These are given by $g=L_{j}$ where $L_{j} \in O$. It is clear that for $L_{r}$ and $L_{s} \in O, g^{e}=L_{r} g L_{s}$; but for $g=L_{j}$ the product $L_{r} g L_{s}=L_{t}$ can only be one of the 24 elements in the $O$ group. Thus the multi- plicity is 24 . Points of this character are points $C$ and $D$ in Fig. 3.

A misorientation which has multiplicity of 6 must be associated with a group $Q_{g}$ which contains cyclic subgroups of order 2 and 3 . This is a consequence of Lagrange's theorem (Hamermesh, 1964) and theorem 5. Point $A$ in Fig. 3 will have a multiplicity of 6 and is formed by the intersection of lines associated with multiplicities of $2[(35)]$ and $3[(36)]$. This misorientation is of the type

$$
g=\left(\begin{array}{ccc}
\frac{2}{3} & \frac{2}{3} & -\frac{1}{3} \\
-\frac{1}{3} & \frac{2}{3} & \frac{2}{3} \\
\frac{2}{3} & -\frac{1}{3} & \frac{2}{3}
\end{array}\right) .
$$

A misorientation which has multiplicity of 8 has been found to contain the cyclic subgroups associated with order 2 and order 4 at the intersection point $E$. This misorientation is of the type

$$
g=\left(\begin{array}{ccc}
1 & 0 & 0 \\
0 & \frac{\sqrt{2}}{2} & \frac{\sqrt{2}}{2} \\
0 & \frac{-\sqrt{2}}{2} & \frac{\sqrt{2}}{2}
\end{array}\right) .
$$

Point $B$ is associated with multiplicity of 4 since it is formed from the intersection of $A B$ and $B E$ which each have multiplicity of 2 . This misorientation is of the type

$$
g=\left(\begin{array}{ccc}
\frac{1}{2} & \frac{1}{2} & \frac{\sqrt{2}}{2} \\
-\frac{1}{2} & -\frac{1}{2} & \frac{\sqrt{2}}{2} \\
\frac{\sqrt{2}}{2} & \frac{-\sqrt{2}}{2} & 0
\end{array}\right)
$$

\subsection{Additional multiplicity due to the inverse property}

To this point we have described the multiplicity occurring in specified misorientations due to rightand left-handed symmetry elements selected from the $O$ point-symmetry group only. Additional multiplicity arises from the misorientation inverse property given in (7). In the previous section the equivalence of misorientations given in (37) and (38) was noted, and attributed to the inverse property. Additional consequences are now described.

First consider the plane $\varphi_{1}=\varphi_{2}$ which is plane $A B C$ in Fig. 3. All misorientations located upon this plane have Euler angles of the form $g=(\varphi, \phi, \varphi)$. From (7) the inverse of such misorientations has the form $g^{-1}=$ $(\pi-\varphi, \phi, \pi-\varphi)$. This inverse point is physically equivalent to $g$ under the action of symmetry elements selected from the $O$ point group:

$$
L_{010}^{2} g^{-1} L_{010}^{2}=g \text {. }
$$


Table 2. CSL boundaries for $\Sigma \leq 49$ ( $m$ is the multiplicity)

\begin{tabular}{|c|c|c|c|c|c|c|}
\hline \multirow[b]{2}{*}{$\Sigma$} & \multirow[b]{2}{*}{$m$} & \multicolumn{3}{|c|}{ Euler angles } & \multicolumn{2}{|c|}{ Axis angle } \\
\hline & & $\varphi_{1}$ & $\phi$ & $\varphi_{2}$ & $\langle h, k, l\rangle$ & $\omega$ \\
\hline 3 & 12 & $45 \cdot 00$ & $70 \cdot 53$ & $45 \cdot 00$ & $1,1,1$ & $60 \cdot 00$ \\
\hline 5 & 8 & 0.00 & $90 \cdot 00$ & $36 \cdot 86$ & $1,0,0$ & $36 \cdot 86$ \\
\hline 7 & 6 & $26 \cdot 56$ & $73 \cdot 40$ & $63 \cdot 44$ & $1,1,1$ & $38 \cdot 21$ \\
\hline 9 & 4 & $26 \cdot 56$ & $83 \cdot 62$ & $26 \cdot 56$ & $1,1,0$ & 38.94 \\
\hline 11 & 4 & $33 \cdot 68$ & $79 \cdot 53$ & $33 \cdot 68$ & $1,1,0$ & $50 \cdot 47$ \\
\hline $13 a$ & 8 & $0 \cdot 00$ & $90 \cdot 00$ & $22 \cdot 62$ & $1,0,0$ & $67 \cdot 38$ \\
\hline $13 b$ & 6 & $18 \cdot 43$ & $76 \cdot 66$ & $71 \cdot 57$ & $1,1,1$ & $27 \cdot 79$ \\
\hline 15 & 2 & 19.65 & $82 \cdot 33$ & $42 \cdot 27$ & $2,1,0$ & $48 \cdot 19$ \\
\hline $17 a$ & 8 & 0.00 & $90 \cdot 00$ & $28 \cdot 07$ & $1,0,0$ & $61 \cdot 93$ \\
\hline $17 b$ & 4 & $45 \cdot 00$ & 86.63 & $45 \cdot 00$ & $1,1,0$ & $86 \cdot 63$ \\
\hline $19 a$ & 4 & $18 \cdot 44$ & $86 \cdot 98$ & $18 \cdot 44$ & $1,1,0$ & $26 \cdot 53$ \\
\hline $19 b$ & 6 & $33 \cdot 69$ & $71 \cdot 59$ & $56 \cdot 31$ & $1,1,1$ & $73 \cdot 17$ \\
\hline $21 a$ & 6 & $14 \cdot 03$ & $79 \cdot 02$ & $75 \cdot 97$ & $1,1,1$ & $21 \cdot 78$ \\
\hline $21 b$ & 2 & $22 \cdot 83$ & $79 \cdot 02$ & $50 \cdot 91$ & $2,1,1$ & $44 \cdot 41$ \\
\hline 23 & 1 & $15 \cdot 25$ & $82 \cdot 51$ & $52 \cdot 13$ & $3,1,1$ & $40 \cdot 45$ \\
\hline $25 a$ & 8 & 0.00 & $90 \cdot 00$ & $16 \cdot 26$ & $1,0,0$ & $16 \cdot 26$ \\
\hline $25 b$ & 6 & $36 \cdot 87$ & 90.00 & $53 \cdot 13$ & $3,3,1$ & 51.68 \\
\hline $27 a$ & 4 & $21 \cdot 80$ & $85 \cdot 75$ & $21 \cdot 80$ & $1,1,0$ & $31 \cdot 59$ \\
\hline $27 b$ & 1 & 15.07 & $85 \cdot 75$ & $31 \cdot 33$ & $2,1,0$ & $35 \cdot 43$ \\
\hline $29 a$ & 8 & 0.00 & $90 \cdot 00$ & $43 \cdot 60$ & $1,0,0$ & $43 \cdot 60$ \\
\hline $29 b$ & 6 & 33.69 & $84 \cdot 06$ & $56 \cdot 31$ & $2,2,1$ & $46 \cdot 40$ \\
\hline $31 a$ & 2 & $27 \cdot 41$ & $78 \cdot 84$ & 43.66 & $3,2,0$ & $54 \cdot 50$ \\
\hline $31 b$ & 6 & $11 \cdot 31$ & $80 \cdot 72$ & 78.69 & $1,1,1$ & $17 \cdot 90$ \\
\hline
\end{tabular}

It follows that every point on plane $A B C$ has an additional multiplicity of 2 .

Next, consider misorientations on the plane $A B D$ in Fig. 3. All such points are of the form $g=$ $(\varphi, \phi, \pi / 2-\varphi)$. The inverse property requires that $g^{-1}=(\varphi+\pi / 2, \phi, \pi-\varphi)$. This inverse point is physically equivalent to point $g$ under the following combination of symmetry operations taken from the $O$ group:

$$
L_{00 \overline{1}}^{4} g^{-1} L_{00 \overline{1}}^{4}=g .
$$

Thus every point on plane $A B D$ increases its multiplicity by a factor of 2 .

For line $C D$ in Fig. 3 all misorientations have the form $g=\left(0, \pi / 2, \varphi_{2}\right)$ and the equivalent inverse misorientations must have the form $g^{-1}=$ $\left(\pi-\varphi_{2}, \pi / 2, \pi\right)$. Again, $g^{-1}$ can be shown to be equivalent to $g$ under

$$
L_{\overline{1} 00}^{4} g^{-1} L_{100}^{4}=g .
$$

The multiplicity of any points on line $C D$ must, consequently, increase by a factor of two. Point $E$ lying on line $C D$ increases in multiplicity from 8 to 16 as a consequence.

With the exception of misorientation points in common with intersecting planes, the multiplicity of points in plane $B C D$ is not altered by the inverse property. As for points on the curved surface $A C D$, it is difficult to prove that there do not exist specific points which are affected by the inverse property, but no general effect occurs except at the intersection with planes $A B C$ and $A B D$. Points $A, B, C$ and $D$

\begin{tabular}{ccrcccc} 
& \multicolumn{3}{c}{ Euler angles } & \multicolumn{2}{c}{ Axis angle } \\
$\Sigma$ & $m$ & $\varphi_{1}$ & $\phi$ & $\varphi_{2}$ & $\langle h, k, l\rangle$ & $\omega$ \\
$33 a$ & 2 & $12 \cdot 34$ & $83 \cdot 04$ & $58 \cdot 73$ & $3,1,1$ & $33 \cdot 56$ \\
$33 b$ & 4 & $37 \cdot 51$ & $76 \cdot 84$ & $37 \cdot 51$ & $1,1,0$ & $56 \cdot 99$ \\
$35 a$ & 1 & $16 \cdot 86$ & $80 \cdot 13$ & $60 \cdot 46$ & $2,1,1$ & $34 \cdot 05$ \\
$35 b$ & 6 & $30 \cdot 96$ & $88 \cdot 36$ & $59 \cdot 04$ & $3,3,1$ & $43 \cdot 23$ \\
$37 a$ & 8 & $0 \cdot 00$ & $90 \cdot 00$ & $18 \cdot 92$ & $1,0,0$ & $18 \cdot 92$ \\
$37 b$ & 1 & $12 \cdot 53$ & $85 \cdot 35$ & $40 \cdot 60$ & $3,1,0$ & $43 \cdot 14$ \\
$37 c$ & 6 & $36 \cdot 87$ & $71 \cdot 08$ & $53 \cdot 13$ & $1,1,1$ & $50 \cdot 57$ \\
$39 a$ & 6 & $21 \cdot 80$ & $75 \cdot 14$ & $68 \cdot 20$ & $1,1,1$ & $32 \cdot 20$ \\
$39 b$ & 1 & $29 \cdot 20$ & $87 \cdot 06$ & $48 \cdot 12$ & $3,2,1$ & $50 \cdot 13$ \\
$41 a$ & 8 & $0 \cdot 00$ & $90 \cdot 00$ & $17 \cdot 68$ & $1,0,0$ & $17 \cdot 68$ \\
$41 b$ & 2 & $17 \cdot 10$ & $84 \cdot 40$ & $36 \cdot 03$ & $2,1,0$ & $40 \cdot 88$ \\
$41 c$ & 4 & $36 \cdot 77$ & $77 \cdot 32$ & $36 \cdot 87$ & $1,1,0$ & $55 \cdot 88$ \\
$43 a$ & 6 & $9 \cdot 46$ & $81 \cdot 98$ & $80 \cdot 54$ & $1,1,1$ & $15 \cdot 18$ \\
$43 b$ & 2 & $12 \cdot 10$ & $87 \cdot 33$ & $24 \cdot 78$ & $2,1,0$ & 27.91 \\
$43 c$ & 4 & $45 \cdot 00$ & $80 \cdot 63$ & $45 \cdot 00$ & $3,3,2$ & $60 \cdot 77$ \\
$45 a$ & 2 & $10 \cdot 30$ & $83 \cdot 62$ & $63 \cdot 44$ & $3,1,1$ & $28 \cdot 62$ \\
$45 b$ & 6 & $26 \cdot 57$ & $83 \cdot 62$ & $63 \cdot 43$ & $2,2,1$ & $36 \cdot 87$ \\
$45 c$ & 6 & $38 \cdot 66$ & $84 \cdot 90$ & $51 \cdot 34$ & $2,2,1$ & $53 \cdot 13$ \\
$47 a$ & 6 & $26 \cdot 56$ & $87 \cdot 56$ & $63 \cdot 44$ & $3,3,1$ & $37 \cdot 07$ \\
$47 b$ & 1 & $22 \cdot 71$ & $82 \cdot 67$ & $35 \cdot 39$ & $3,2,0$ & $43 \cdot 66$ \\
$49 a$ & 6 & $30 \cdot 96$ & $72 \cdot 17$ & $59 \cdot 04$ & $1,1,1$ & $43 \cdot 57$ \\
$49 b$ & 1 & $10 \cdot 62$ & $85 \cdot 32$ & $47 \cdot 49$ & $5,1,1$ & $43 \cdot 57$ \\
$49 c$ & 2 & $30 \cdot 35$ & $75 \cdot 82$ & $49 \cdot 27$ & $3,2,2$ & $49 \cdot 23$
\end{tabular}

increase in multiplicity by a factor of 2 due to the inverse property.

\section{CSL boundaries in the asymmetric domain}

The coincidence site lattice (CSL) theory (Bollmann, 1970; Brandon, 1966) is frequently considered to provide a basis for 'specialness' in grain boundary properties. Coincidence refers to certain special misorientations between two crystal lattices which form a coincidence superlattice when their lattice points are permitted to interpenetrate each other. The parameter $\Sigma$ is inversely related to the fraction of lattice sites which are coincident between these two interpenetrating lattices. In adjacent grains of special misorientation and low $\Sigma$ number it is possible to form a grain boundary exhibiting a high degree of atomic congruence and consequently low energy and improved resistance to intercrystalline fracture. Some evidence supporting this point of view has been reported in recent literature (Don \& Majumdar, 1986; Watanabe, 1983; Lim \& Raj, 1984; Zhao \& Adams, 1986). In the interest of completeness this section will relate these special CSL boundaries to the asymmetric domain in the space of Euler angles.

For $\Sigma \leq 49$ the complete list of CSL boundaries given by Grimmer, Bollmann \& Warrington (1974) has been converted from axis-angle pairs to the triplet of Euler angles $\left(\varphi_{1}, \phi, \varphi_{2}\right)$ in the asymmetric subvolume using the equations provided by Bunge (1982) [his equations (2.50) and (2.62)]. This listing is given in Table 2 and all the CSL boundaries are carefully located in Fig. 4. It has been noted that all the CSL boundaries lie on the surface of the asymmetric subvolume except $\Sigma 39 b$. 


\section{Summary and discussion}

A comprehensive derivation for a new asymmetric domain for intercrystalline misorientation in cubic materials has been presented in $\S 2$. This new domain is represented in the space of Euler angles, and exhibits significant advantages over the previous domain defined by MacKenzie $(1958,1964)$ using the axis-angle parameters. The chief advantage is that the invariant measure associated with random distributions of misorientation is nearly constant. [The range of variation for the invariant measure in the new domain is $0.943 \leq \sin \phi \leq 1.000$, in contrast to the variation in MacKenzie's domain which is $0 \leq$ $\sin \Theta \sin ^{2}(\omega / 2) \leq 0.271$.] For small values of the rotation angle in MacKenzie's domain serious ambiguity exists in the rotation axis. This problem is not present in the new domain defined by this paper. A further advantage is the simplicity of definition possible for the new domain in the space of Euler angles. This is typified by the definition given in (30) and (31). Finally, the new domain is the first to be discovered in the space of Euler angles. Whereas previous descriptions of distribution functions were given over multiples of the asymmetric domain, the new domain will significantly reduce the computation time required to represent such functions, and significantly increase the clarity of their representation.

In $\S 3$ a detailed group-theoretical treatment of the problem of multiplicity has been presented. It was shown that all possible misorientations exhibiting multiplicities of $m>1$ can be classified into a finite number of types. These types were shown to exist only on the surface of the asymmetric domain. Their multiplicities were shown to be 2, 4, 6, 8, 12, 16 and 48 when the inverse property of (7) was included. With respect to the asymmetric domain, all possible misorientations exhibiting multiplicities of $m>1$ have been identified in Fig. 3. This problem of multiplicity was previously treated in conjunction with the

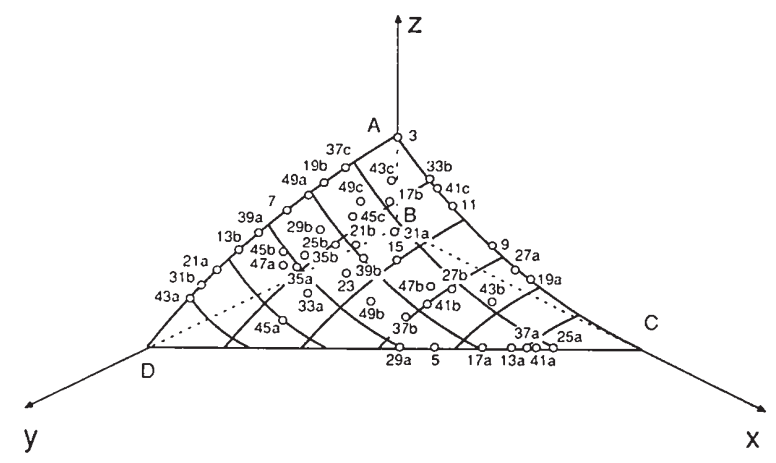

Fig. 4. The positions of all CSL boundaries in the asymmetric region for $\Sigma \leq 49$. The coordinates are defined by: $x=$ $(\pi / 4) \sqrt{2}-\sqrt{2} \varphi_{1}-\left(\varphi_{2}-\varphi_{1}\right) / \sqrt{2} ; y=\left(\varphi_{2}-\varphi_{1}\right) / \sqrt{2} ; z=\pi / 2-\phi$. orientation distribution function (ODF) (Bunge, 1982; Hansen, Pospiech \& Lucke, 1978), but the present paper is the first to treat the problem thoroughly for cubic misorientations. Identifying all such multiplicities is a very important aspect in interpreting distribution functions of the misorientation.

$\S 4$ provides an important link with the present work to the geometrical theory of grain boundaries. Table 2 lists the locations for all CSL boundaries for $\Sigma \leq 49$ in the new asymmetric domain. Further, their associated multiplicity have been identified. This table should provide an important bridge between theorists and experimentalists interested in the properties of individual boundaries and those interested in measuring and describing their distributions in polycrystalline media. Clearly, both aspects are important in developing models for macroscopic behavior of these materials.

The authors wish to acknowledge the support of the United States Department of Energy, Office of Basic Energy Sciences, under grant No. DE-FG0285ER45203. Critical review by Professor Dorian Hatch of the Physics Department at Brigham Young University is gratefully acknowledged.

\section{References}

AdAMS, B. L. (1986). Metall. Trans. A17, 2199-2207.

BollmanN, W. (1970). Crystal Defects and Crystalline Interfaces. New York: Springer-Verlag.

Brandon, D. G. (1966). Acta Metall. 14, 1479.

BuNGE, H. J. (1982). Texture Analysis in Materials Science. London: Butterworth.

Don, J. \& MAJUMdAR, S. (1986). Acta Metall. 34, 961-967.

Fraleigh, J. B. (1982). A First Course in Abstract Algebra. Reading, MA: Addison-Wesley.

Grimmer, H., BollmanN, W. \& Warrington, D. H. (1974). Acta Cryst. A30, 197-207.

Haessner, F., Pospiech, J. \& Sztwiertnia, K. (1983). Mater. Sci. Eng. 57, 1-15.

HAMERMESH, M. (1964). Group Theory. Reading, MA: AddisonWesley.

Hansen, J., Pospiech, J. \& Lucke, K. (1978). Tables for Texture Analysis of Cubic Crystals. Berlin: Springer-Verlag.

Harase, J., Shimizu, R., Kuroki, K., Nakayama, T., Wada, T. \& WATANABE, T. (1986). Grain Boundary Structure and Related Phenomena. Proceedings of JIMIS-4, pp. 563-570.

Harase, J., Shimizu, R. \& Watanabe, T. (1986). Annealing Processes: the Effects of Grain Boundary Character Distribution on Grain Growth and Texture Formation in $\mathrm{Fe}-3 \% \mathrm{Si}$. Proc. 7th Risø Int. Symp. Metall. Mater. Sci., pp. 343-348, edited by N. HANSEN et al. Ris $\varnothing$ National Laboratory, Roskilde, Denmark.

LIM, L. C. \& RAJ, R. (1984). Acta Metall. 32, 1177-1181.

MACKENZIE, J. K. (1958). Biometrika, 45, 229-240.

MacKenzie, J. K. (1964). Acta Metall. 12, 223-225.

Neumann, P. \& Toennessen, A. (1987). Proc. 3rd Int. Conf. Fatigue and Fatigue Thresholds, Charlottesville, VA, USA.

Pospiech, J., GnAtek, A. \& Fichtner, K. (1974). Krist. Tech. 9, 729-742.

Pospiech, J., Sztwiertnia, K. \& HAessner, F. (1986). Texture Microstruct. 6, 201-215.

Watanabe, T. (1983). Metall. Trans. A14, 531. 
ZhaO, J. \& Adams, B. L. (1986). Proc. Int. Conf. Fatigue, Corrosion Cracking, Fracture Mechanics, and Failure Analysis: The Mechanism of Fracture, edited by V. S. GoEL, pp. 597-603. Metals Park, Ohio: American Society for Metals.
ZhaO, J., AdAms, B. L. \& MorRis, P. R. (1988). Textures Microstruct. In the press.

ZhaO, J., KoONTZ, J. S. \& AdAms, B. L. (1988). Metall Trans. In the press. 